\title{
miR-127 suppresses gastric cancer cell migration and invasion via targeting Wnt7a
}

\author{
LINLIN WANG $^{1}$, XUFEI WANG ${ }^{2}$ and XUEFENG JIANG ${ }^{3}$ \\ ${ }^{1}$ Ultrasound Department of China-Japan Union Hospital, Jilin University, Changchun, Jilin 130033; \\ ${ }^{2}$ Department of Opthalmology, Jilin Province People's Hospital, Changchun, Jilin 130021; \\ ${ }^{3}$ Gastroenterology Department of China-Japan Union Hospital, Jilin University, Changchun, Jilin 130000, P.R. China
}

Received January 4, 2018; Accepted January 4, 2019

DOI: $10.3892 / 01.2019 .9955$

\begin{abstract}
Gastric cancer (GC) is a malignant tumor originating from the mucosal epithelium of the stomach. Patients suffering from this disease may have occurrence of residual GC due to delay in diagnosis and treatment. In addition, abnormal expression of microRNAs (miRNAs) is involved in GC progression. Therefore, we examined the underlying mechanism of miR-127 in GC. The expression of miR-127 and Wnt7a was examined in GC using RT-qPCR and western blot analysis. A Transwell assay was used to assess the ability of GC cell migration and invasion. Luciferase reporter assay was used to verify the specific target of miR-127 in GC. The results showed miR-127 expression was lower in GC than normal samples, while Wnt7a expression was detected at a higher level in GC than normal samples. The association between miR-127 and Wnt7a expression was negatively correlated in GC tissues. miR-127 mimic in the two GC cell lines markedly curbed cell migration and invasion, while inhibition of miR-127 showed the opposite effect. In addition, Wnt7a siRNA significantly inhibited GC cell migration and invasion and Wnt7a was verified as a specific target of miR-127 in GC cells. Wnt7a reversed the ability of GC cell migration and invasion regulated by miR-127. In conclusion, miR-127 could curb GC cell migration and invasion by upregulating Wnt7a, indicating its potential application in GC diagnosis and therapy.
\end{abstract}

\section{Introduction}

Gastric cancer (GC) is a malignant tumor originating from the mucosal epithelium of the stomach. The incidence of GC ranks first compared to other types of malignant tumors in China $(1,2)$. According to the clinical data, patients may have occurrence of residual GC due to delay in diagnosis and

Correspondence to: Dr Xuefeng Jiang, Gastroenterology Department of China-Japan Union Hospital, Jilin University, 126 Xiantai Street, Changchun, Jilin 130000, P.R. China

E-mail: eif387127@163.com

Key words: miR-127, migration, invasion, gastric cancer, Wnt7a treatment. Residual GC is more aggressive than common GC and is difficult to identify in the early stage (3). Most GC patients are diagnosed at an advanced stage. Thus, the early diagnosis rate of GC in China remains low. Although there have been great clinical efforts to treat GC, the disease remains a major clinical burden. Therefore, exploring the mechanism of the GC progression is necessary.

Mounting evidence has shown that microRNAs (miRNAs) play important roles in the progression of various types of cancer including GC by targeting several mRNA genes (4-6). Most of the miRNAs are verified as potential therapeutic targets for treating GC. For instance, miR-320a acts as a suppressor of GC cell growth by regulating ADAM10 (7). miRNA-3978 was also identified to suppress GC metastasis by regulating PCBP1 (8). However, miR-214 facilitated the cell viability and migration of GC by inhibiting A2AR and PRDM16 (9). miR-324 enhanced the progression of GC by regulating Smad4 (10). Thus, previous studies on microRNAs are imperative in the treatment of GC and provide a deeper understanding of the regulation mechanism of GC.

Previous findings have shown that miR-127 played an important role in cell growth, invasion and metastasis of various cancer types. miR-127 was reported to be a tumor suppressor involved in the regulation of cell proliferation, invasion of ovarian cancer and cell cycle progression of pancreatic cancer by targeting BAG5 $(11,12)$. A recent study identified the inhibition of miR-127 on osteosarcoma cell proliferation and migration (13). In addition, miR-127 acted as a tumor promoter in regulating cell migration and invasion of glioblastoma through downregulation of SEPT7 (14). Nevertheless, there are few reports on the biological mechanism of miR-127 in GC.

Wnt is a highly conserved secreted glycoprotein playing an important role in the regulation of cell proliferation, migration and tumorigenesis through the Wnt/ $\beta$-catenin signaling pathway (15-17). Wnt-7a is encoded by the WNT7A gene. A recent study stated that Wnt7a played different roles in the invasion and metastasis of various tumors (18-20), but whether the role of Wnt7a is tumor promotion or inhibition is contradictory. Wnt7a was proven to be overexpressed in ovarian cancer and to function as a tumor promoter in regulating ovarian cancer development (21). Ramos-Solano et al corroborated that Wnt7a was downregulated in cervical cancer and re-expression of Wnt7a inhibited cell proliferation and 
migration (22). However, the role Wnt7a plays in GC progression regulated by miR-127 remains unclear.

We studied the effect of miR-127 in GC development and the biological mechanism of miR-127 in regulation of GC cell migration and invasion. We discovered a new miRNA, miR-127, acted as a GC tumor suppressor. miR-127 mimic suppressed GC cell migration and invasion and reduced Wnt7a expression, while miR-127 silencing had the opposite effect. Furthermore, we demonstrated the negatively correlation between miR-127 and Wnt7a expression in GC tissues. Therefore, our results indicated that the role of $\mathrm{miR}-127 / \mathrm{Wnt} 7 \mathrm{a}$ in GC migration and invasion was important, proving a new idea for GC treatment.

\section{Materials and methods}

Tumor tissues. Twenty tumor tissues were obtained from GC patients who underwent surgery at the China-Japan Union Hospital, Jilin University (Changchun, China) after signing written consent. The study was approved by the Ethics Committee of Jilin University. The collected tissues were immediately stored at $-80^{\circ} \mathrm{C}$.

Cell culture. All the GC cell lines (AGS, CES-1, BGC-823 and HGC-27) were purchased from the Shanghai Institute of Cell Biology of the Chinese Academy of Sciences. The tumor cell lines were cultured in RPMI-1640 medium (Invitrogen; Thermo Fisher Scientific, Inc., Waltham, MA, USA) containing $10 \%$ fetal bovine serum (FBS; Gibco; Thermo Fisher Scientific, Inc.), penicillin (100 U/ml) and streptomycin (100 $\mu \mathrm{g} / \mathrm{ml})$ (Solarbio, Beijing, China) and incubated at $37^{\circ} \mathrm{C}$ under $5 \% \mathrm{CO}_{2}$ atmosphere.

Cell transfection. A miR-127 mimic was transfected into GC cells to overexpress the miR-127 or miR-127 inhibitor to knock down the miR-127. Synthetic miR-127 mimic, miR-127 inhibitor and control were obtained from GenePharma (Shanghai, China). BGC-823 and HGC-27 cells used in this study were placed into 24 -well plates $24 \mathrm{~h}$ before transfection. The Lipofectamine $2000^{\mathrm{TM}}$ reagent (Invitrogen; Thermo Fisher Scientific, Inc.) was used in the transfection into GC cell lines. All procedures of the transfection were performed following the manufacturer's instructions. The transfected cells were divided into several groups: control, miR-127 mimic and miR-127 inhibitor; con siRNA and Wnt7a siRNA; control mimic + control vector, miR-127 mimic + control vector and miR-127 mimic + Wnt7a vector. After transfection for $48 \mathrm{~h}$, cells were collected for subsequent experimentation.

$R T-q P C R$. Total RNA was extracted from GC cells and tissues using TRIzol reagent (Invitrogen; Thermo Fisher Scientific, Inc.). RT-qPCR was conducted by TaqMan PCR kit (Takara, Dalian, China) following the manufacturer's instructions. SYBR Premix ExTaq II (Takara) was used to perform quantitative PCR. The primer sequences used were: miR-127-F, GGAAGATCT GTAGTCCTGTCTGTTGGTCAG and miR-127-R, CCCAAG CTTCCTGAAGAACTGCTTCCGCC; Wnt7a-F, GTAGTT CGGCGTCGTTTTAC and Wnt7a-R, CGAAACCGTCTA TCGATACG; U6-F, CTCGCTTCGGCAGCACA and U6-R, AACGCTTCACGAATTTGCGT; GAPDH-F, TGGTATCGT
GGAAGGACTC and GAPDH-R, AGTAGAGGCAGGGATG ATG. The reactions were performed at $95^{\circ} \mathrm{C}$ for $10 \mathrm{~min}$, followed by 40 cycles of $95^{\circ} \mathrm{C}$ for $15 \mathrm{sec}$ and $58^{\circ} \mathrm{C}$ for $1 \mathrm{~min}$, and a dissociation stage at $60^{\circ} \mathrm{C}$ for $10 \mathrm{~min}$. GAPDH and U6 were used as an internal control. Analysis of relative gene expression data was made using RT-qPCR and the $2^{-\Delta \Delta \mathrm{Cq}}$ method reported by Livak and Schmittgen (23).

Western blot assay. RIPA lysis buffer containing proteinase inhibitors (Beyotime Institute of Biotechnology, Haimen, China) were used to extract total protein from the GC cells or tissues. Protein concentration was measured using BCA reagent kit (Beyotime Institute of Biotechnology). Total protein $(50 \mu \mathrm{g})$ from each group was separated by SDS-PAGE. After electrophoresis, the proteins were transferred to an $\mathrm{NC}$ membrane (Millipore, Billerica, MA,USA). Skim milk (5-10\%) was then used to block the membranes at room temperature for $2 \mathrm{~h}$. Subsequently, the primary antibodies (rabbit polyclonal anti-Wnt7a, cat. no ab100792, 1:500; Abcam, Cambridge, UK; rabbit monoclonal anti-GAPDH, cat. .no. 5174, 1:2,000; Cell Signaling Technology, Inc., Danvers, MA, USA) were added to incubate the proteins at $4^{\circ} \mathrm{C}$ overnight and the goat anti-rabbit peroxidase-conjugated secondary antibodies (cat. no. ab205718, 1:2,000; Abcam) were added for $2 \mathrm{~h}$ at room temperature, respectively. Finally, the enhanced chemiluminescence kit (ECL; Millipore) was used to detect the signals. Densitometric analysis of bands was performed using ImageJ software (National Institutes of Health, Bethesda, MD, USA). GADPH served as a loading control.

Dual luciferase reporter assay. The relative luciferase ability was performed using the recombinant pMIR-reportor luciferase vector. The wild-type and mut-type miR-127 putative targets on Wnt7a 3'-UTR were constructed downstream of pMIR-reporter luciferase vector. We used Lipofectamine 2000 to transfect GC cells with control mimic and miR-127 mimic. The Dual Luciferase Reporter Assay System (Promega Corporation, Madison, WI, USA) was used to measure the luciferase activity values.

Transwell assay. Cell migratory and invasive ability was performed using Transwell assay. For the migration assay, the Transwell chamber with $8 \mu \mathrm{m}$ pore size polycarbonic membrane (Costar, Corning, NY, USA) was firstly placed into the 24-well plates to separate the top and the lower chambers. Secondly, GC cells $\left(1 \times 10^{5}\right)$ with different transfection were seeded into the top chamber, and RPMI-1640 medium containing $20 \%$ FBS was added into the lower chambers as an attractant and then incubated for $24 \mathrm{~h}$ at $37^{\circ} \mathrm{C}$. The cells in the upper chambers subsequently migrated into the lower chamber. Then the migratory cells were stained with $0.1 \%$ crystal violet for $30 \mathrm{~min}$. Images of the migrated cells were captured under a microscope (Zeiss AG, Oberkochen, Germany). For invasion assay, the filter in the upper chamber was coated with Matrigel, otherwise it was similar to the Transwell migration assay.

Statistical analysis. Experiments were repeated in triplicate, SPSS v.19.0 software was used to perform statistical analyses and GraphPad Prism 5.02 software used to complete graph presentations. TargetScan (http://www.targetscan.org/vert_72/) 
A

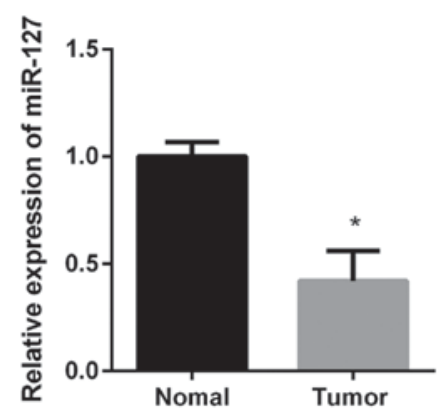

C

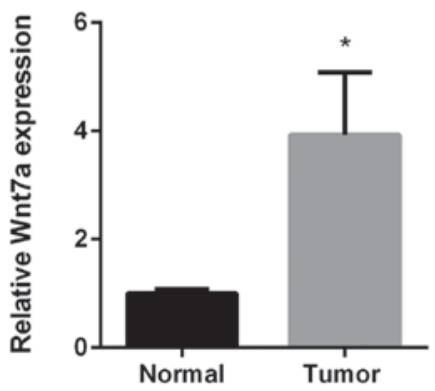

E

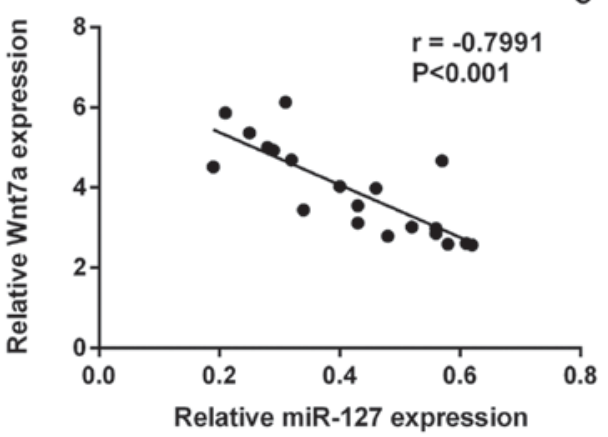

B

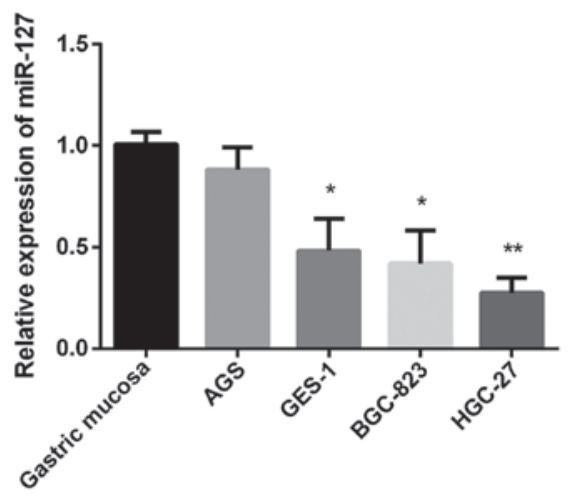

D

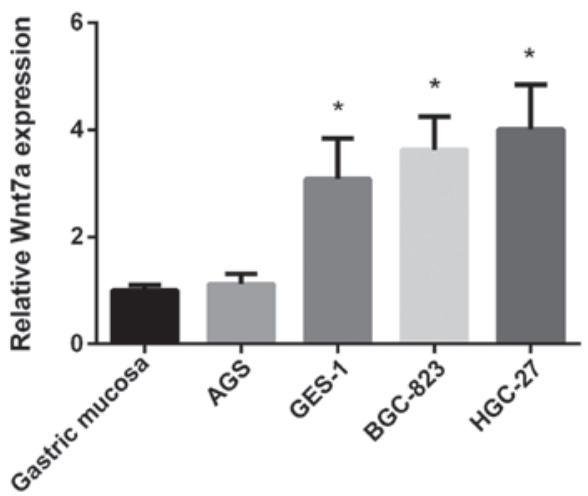

Figure 1. Detection of miR-127 and Wnt7a expression in gastric cancer (GC). (A) RT-qPCR analysis of miR-127 mRNA expression in GC tissues (n=20). (B) RT-qPCR analysis of miR-127 mRNA expression in the four GC cell lines. (C) RT-qPCR analysis of Wnt7a in GC tissues (n=20). (D) RT-qPCR analysis of Wnt7a in the four GC cell lines. (E) Regression analysis of miR-127 and Wnt7a expression in GC tissues $(\mathrm{n}=20)(\mathrm{r}=-0.7991, \mathrm{P}<0.001)$. ${ }^{*} \mathrm{P}<0.05$; ${ }^{* * *} \mathrm{P}<0.01$.

and MiRanda (microrna.org/microrna/home.do) databases were utilized to forecast the target genes of miR-127. Results are represented as the mean $\pm \mathrm{SD}$, and the data were evaluated using Student's t-test or Tukey's post hoc test after ANOVA, with statistically significant difference considered at $\mathrm{P}<0.05$.

\section{Results}

Decreased miR-127 expression and increased Wnt7a expression in GC. First, we investigated miR-127 expression in 20 paired GC tissues and normal tissues using RT-qPCR. miR-127 expression was lower in GC tissues (Fig. 1A). Then, we investigated miR-127 expression in four GC cell lines using RT-qPCR. As shown in Fig. 1B, miR-127 expression was slightly reduced in AGS cell line but significantly decreased in the remaining GC cell lines. The Wnt7a expression in the same 20 paired GC tissues and the four GC cell lines was also examined (Fig. 1C and D). Wnt7a expression in GC tissues was higher than normal tissues, and Wnt7a expression in all GC cell lines was increased. Regression correlation analysis was used to determine the association between miR-127 and Wnt7a expression (Fig. 1E), and the inverse correlation coefficient was $r=-0.7991$. The results indicated that miR-127 inhibited GC progression by upregulating Wnt7a expression.

miR-127 inhibits GC migratory and invasive ability. We used Transwell assay to investigate the ability of GC cell migration and invasion regulated by miR-127. RT-qPCR was used to analyze the relative miR-127 expression in two GC cell lines following transfection with miR-127 mimic and inhibitor (Fig. 2A). Fig. 2B results indicated that miR-127 mimic group showed decreased migration, whereas, the miR-127 inhibitor group enhanced migration in the two cell lines. Overexpression of miR-127 decreased cell invasion, while miR-127 inhibitor promoted cell invasion (Fig. 2C).

Wnt7a promotes GC migratory and invasive ability. We also used Transwell assay to investigate the ability of GC 
A

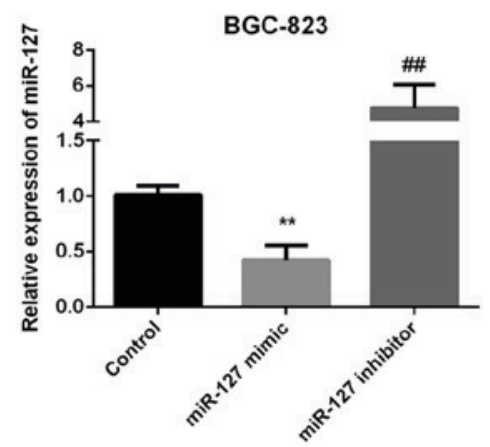

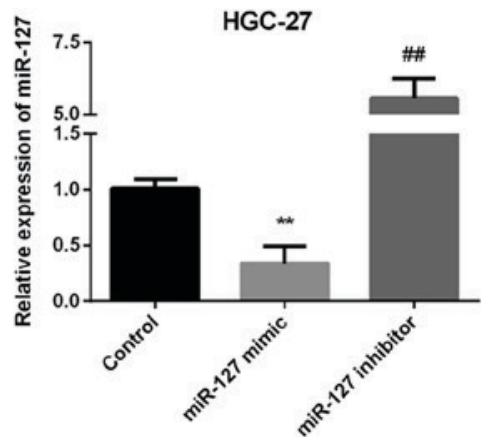

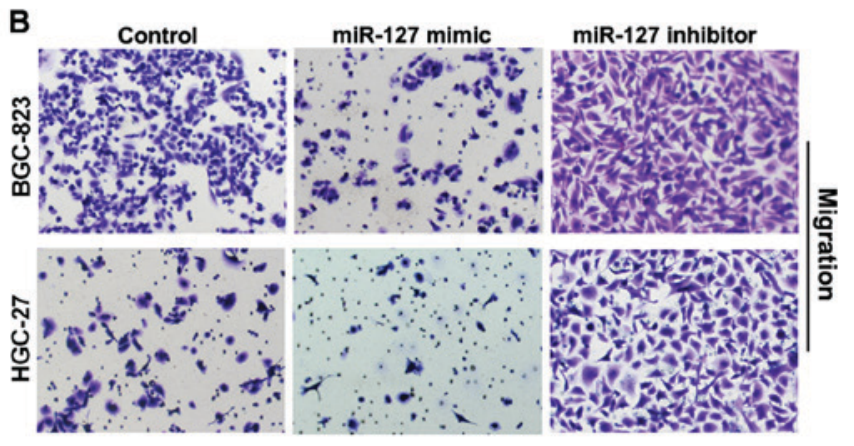

C

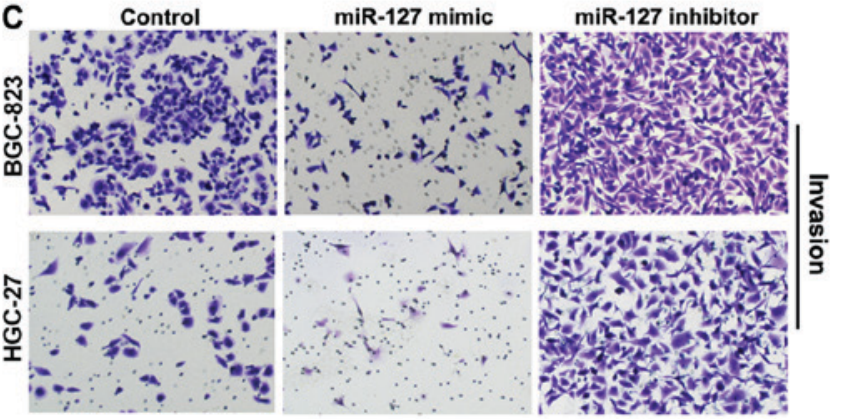

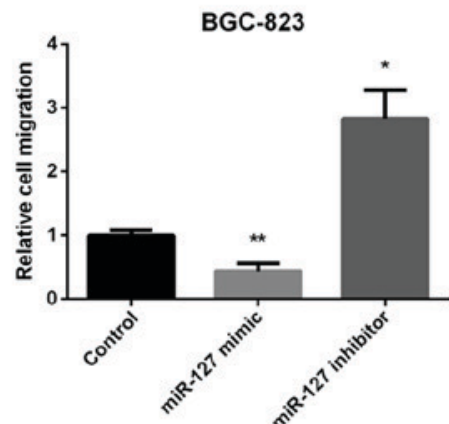
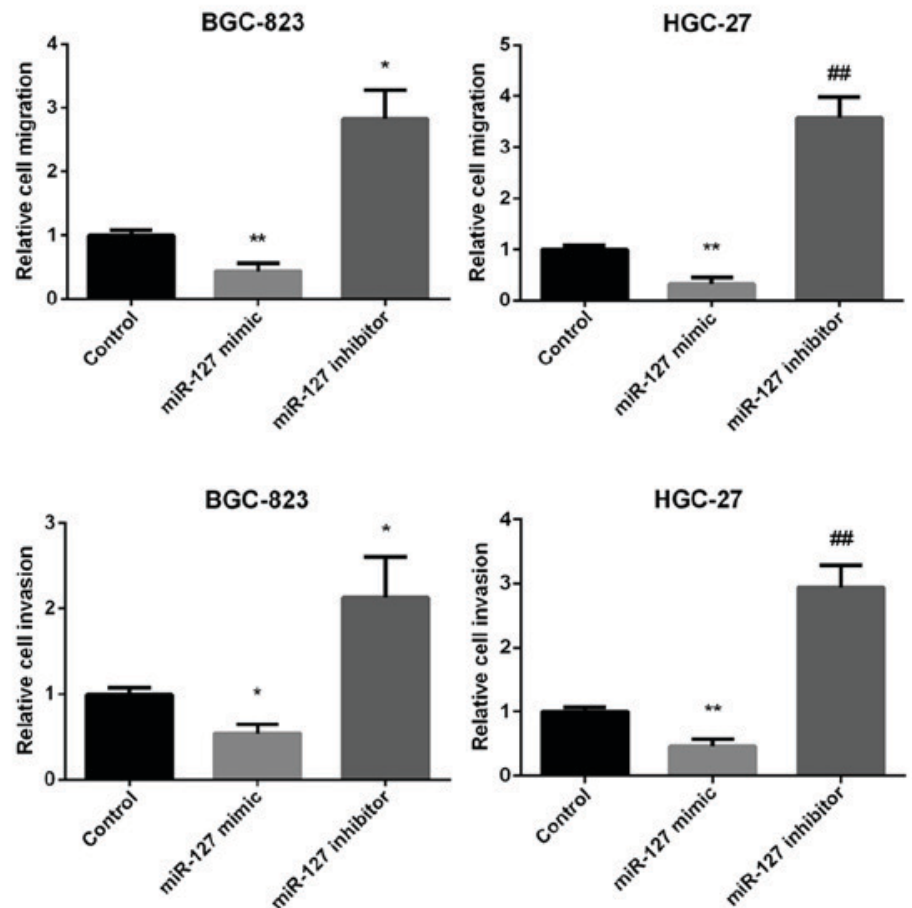

Figure 2. Inhibition of miR-127 in regulation of gastric cancer (GC) cell migration and invasion. (A) Detection of miR-127 expression after transfection with miR-127 mimic and inhibitor in two GC cell lines using RT qPCR. (B) Images and quantitative cell migration in GC cells after transfection with miR-127 mimic and inhibitor. (C) Images and quantitative invasion in $\mathrm{GC}$ cells after transfection with miR-127 mimic and inhibitor. ${ }^{*} \mathrm{P}<0.05$; ${ }^{* *} \mathrm{P}<0.01$; ${ }^{\# \prime} \mathrm{P}<0.01$.

cell migration and invasion regulated by Wnt7a. RT-qPCR and western blot analysis were used to determine the relative Wnt7a expression in two GC cell lines after silencing Wnt7a (Fig. 3A and B). Fig. 3C results show that Wnt7a siRNA group decreased migration in both cell lines compared with control group. In addition, knockdown of Wnt7a decreased cell invasion (Fig. 3D).

Corroboration of Wnt7a as a target of miR-127. It has been reported that miRNA regulation of gene expression via targeting the 3'-UTR of mRNA was very important. Therefore, we used TargetScan and miRanda to verify the direct target of miR-127 and found Wnt7a may be the target of miR-127. The predicted target sites between miR-127 and the Wnt7a are shown in Fig. 4A. Then, we used dual luciferase reporter assay to detect the predicted sequence binding sites of miR-127 and Wnt7a in the BGC-823 and HGC-27 cell lines. The luciferase reporter activity in the miR-127 mimic group was obviously lower than the control group in the two cell lines (Fig. 4B). Then, we detected miR-127 binding ability in mutated type of miR-127. The results stated that the miR-127 mimic group had no effect on the luciferase reporter activity. The results showed that miR-127 inhibited Wnt7a translation by binding to the 3'-UTR of the Wnt7a. We then evaluated the Wnt7a expression in two GC cell lines after transfection with miR-127 mimic or inhibitor. It was shown that Wnt7a mRNA expression and protein level was markedly reduced after overexpression of miR-127 but significantly increased when silencing miR-127 in GC cells (Fig. 4C and D). The results suggested that miR-127 regulated Wnt7a expression by controlling the development of GC.

Reversal of Wnt7a in miR-127 inhibition effect in GC. We used Transwell assay to investigate the role of Wnt7a in GC cell migration and invasion regulated by miR-127. The miR-127 mimic group showed decreased migration compared to the control group in GC cells. However, the re-expression of both miR-127 and Wnt7a showed higher migration compared to 
A

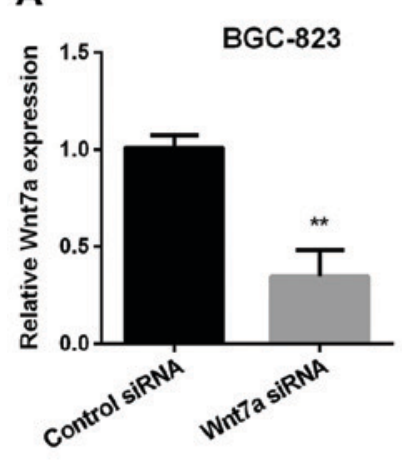

HGC-27

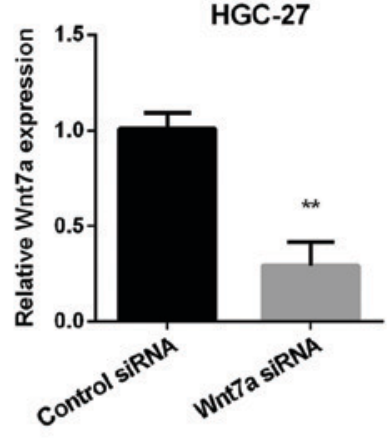

B

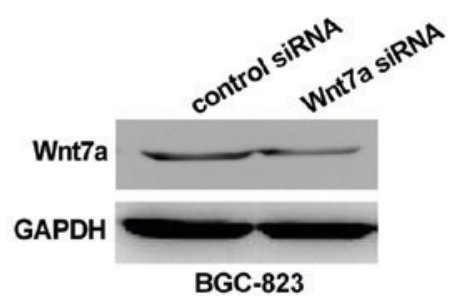

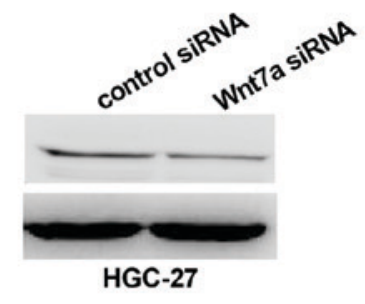

\section{C}

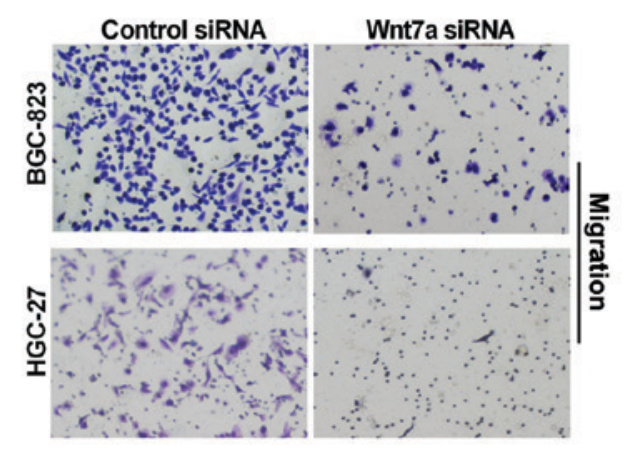

D

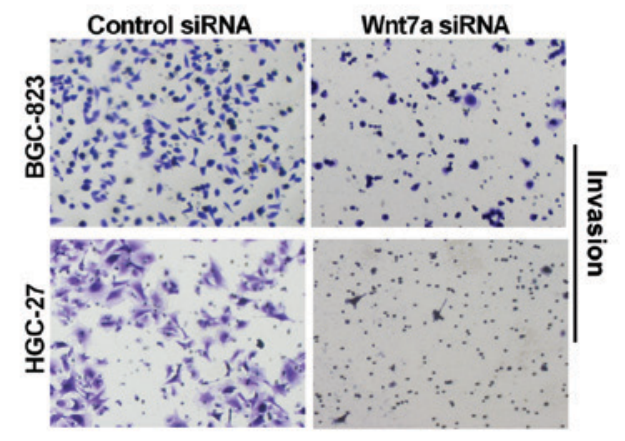

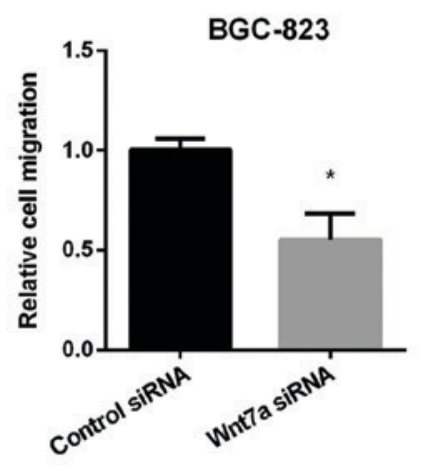
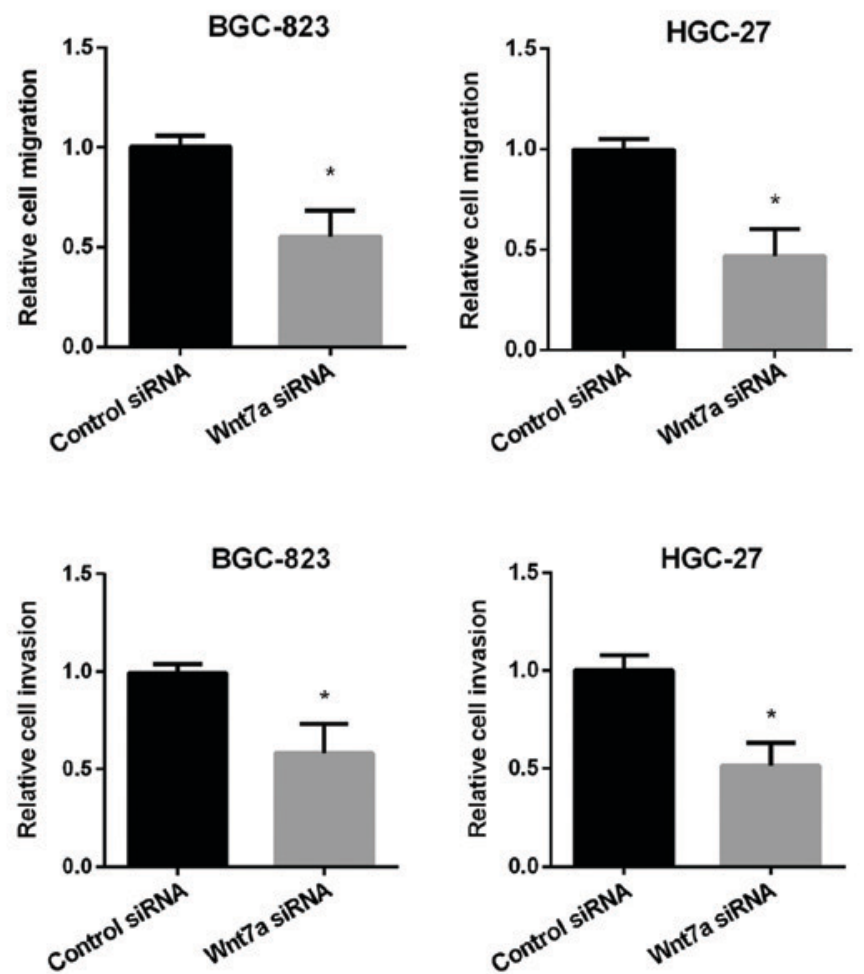

Figure 3. Inhibition of Wnt7a siRNA in the regulation of gastric cancer (GC) cell migration and invasion. (A) Detection of Wnt7a expression after transfection with Wnt7a siRNA in two GC cell lines using RT-qPCR. (B) Detection of Wnt7a protein level after transfection with Wnt7a siRNA in GC cells using western blot analysis. (C) Images and quantitative cell migration in GC cells after transfection with Wnt7a siRNA. (D) Images and quantitative cell invasion in GC cells after transfection with Wnt7a siRNA. ${ }^{*} \mathrm{P}<0.05 ;{ }^{* *} \mathrm{P}<0.01$

cell overexpression of miR-127 alone (Fig. 5A), suggesting that Wnt7a attenuated the inhibitory effect of miR-127 on GC cell migration. In addition, Fig. $5 \mathrm{~B}$ results show that the relative cell invasion in GC cells was decreased in miR-127 mimic group. However, re-expression of both miR-127 and Wnt7a showed higher invasion than cell overexpression of miR-127 alone (Fig. 5B), suggesting that Wnt7a attenuated the inhibitory effect of miR-127 on GC cell invasion. In conclusion, miR-127 inhibited GC cell migration and invasion by targeting Wnt7a.

\section{Discussion}

It has been proven that miR-127 abnormal expression in various malignancies is involved in the development and progression of multiple tumors, including GC (24-27). Our study showed a markedly decreased miR-127 expression in GC, and
miR-127 mimic suppressed GC cell migration and invasion, while miR-127 inhibitor promoted it, which was consistent with previous findings showing that miR-127 decreased in GC and miR-127 mimic inhibited GC cell progression (28).

It is well known that Wnt7a signaling is involved in cancer progression (29). However, the manner in which Wnt genes are regulated in tumors are rarely reported. Recently, Wnt7a was reported to be overexpressed in colorectal cancer and pancreatic cancer (20). We found that Wnt7a expression was obviously higher in $\mathrm{GC}$, which is consistent with the reports that Wnt7a was upregulated in GC (20,30). Previous results also showed that Wnt7a promoted cell proliferation and adhesion regulated by $\mathrm{miR}-15 \mathrm{~b}$, and miR-15b exhibited significant inverse correlation with Wnt7a in ovarian cancer (21). Kim et al found that Wnt7a expression was directly regulated by miR-199a in cutaneous squamous cell carcinoma (31). The present study found 
A

\begin{tabular}{|c|c|}
\hline & $\begin{array}{l}\text { Predicted consequential pairing of target region (top) } \\
\text { and miRNA (bottom) }\end{array}$ \\
\hline $\begin{array}{l}\text { osition } 367-373 \text { of WNT7A } 3 \text { UTR } \\
\text { sa-miR-127-3p }\end{array}$ & 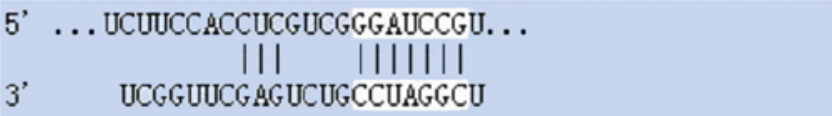 \\
\hline
\end{tabular}

B
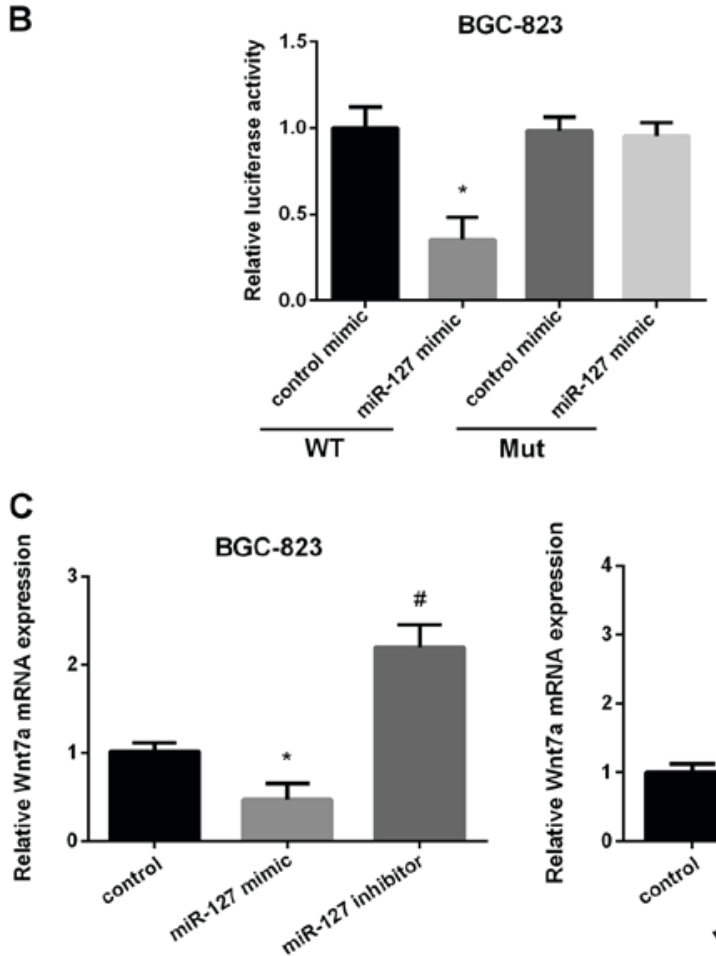

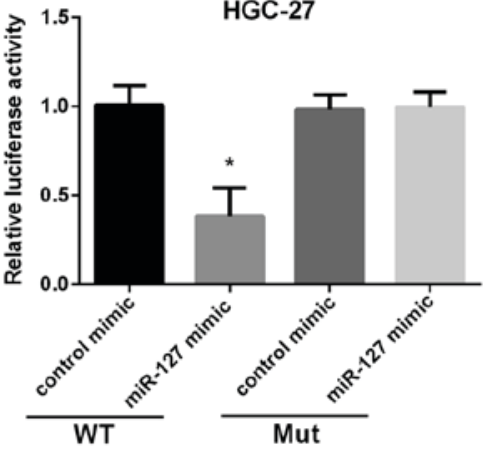

HGC-27

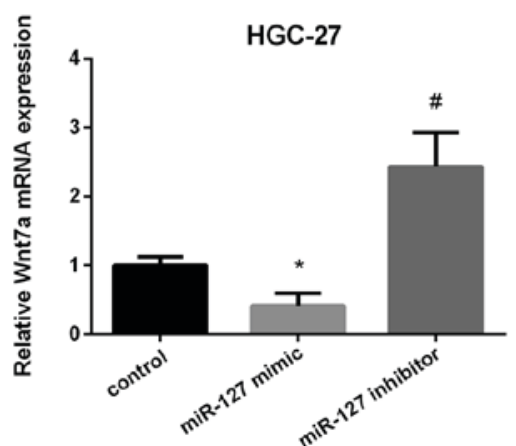

D

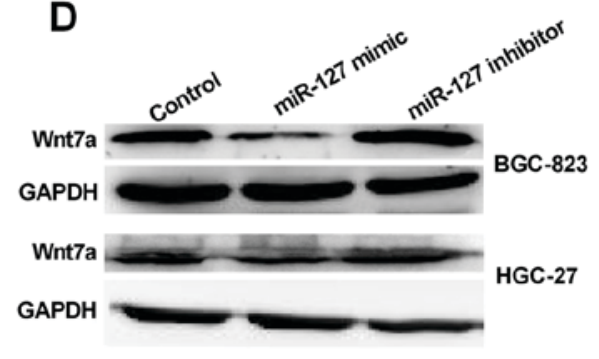

Figure 4. Corroboration of Wnt7a as a direct target of miR-127. (A) Schematic diagram of the binding sites of miR-127 with the 3'-UTR of Wnt7a. (B) Relative luciferase activities in two gastric cancer (GC) cell lines after transfection with miR-127 mimic in wild-type or mutant type. (C) Analysis of Wnt7a mRNA expression in GC cells after transfection with Wnt7a siRNA by RT-qPCR. (D) Analysis of Wnt7a protein level in GC cells after transfection with Wnt7a siRNA evaluated by western blot analysis. ${ }^{*} \mathrm{P}<0.05 ;{ }^{*} \mathrm{P}<0.05$.
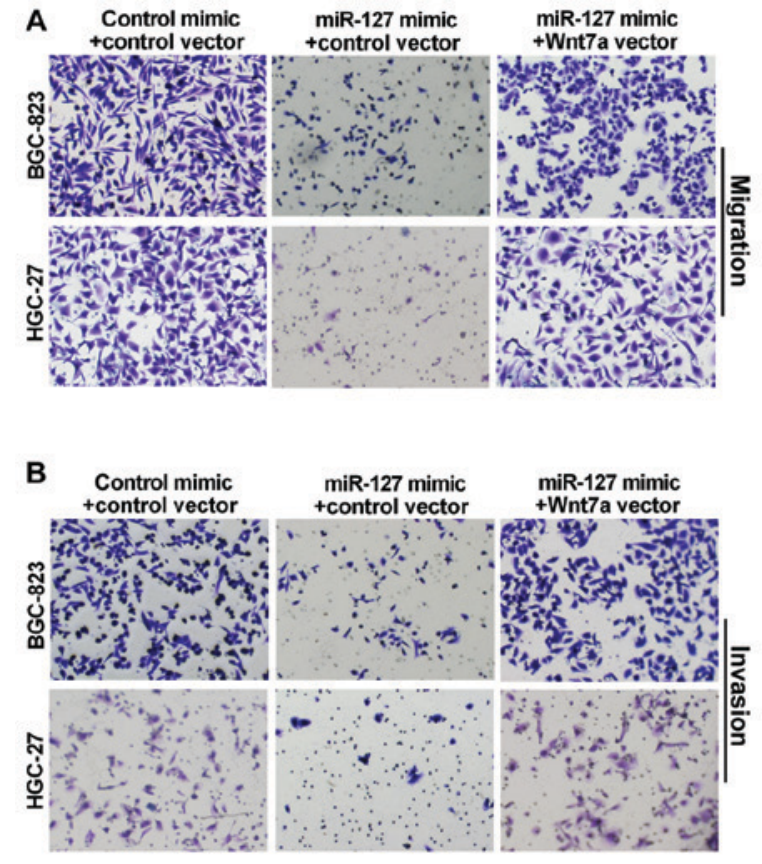

BGC-823

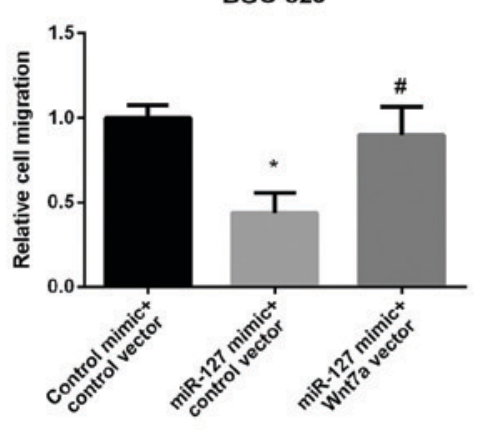

BGC-823

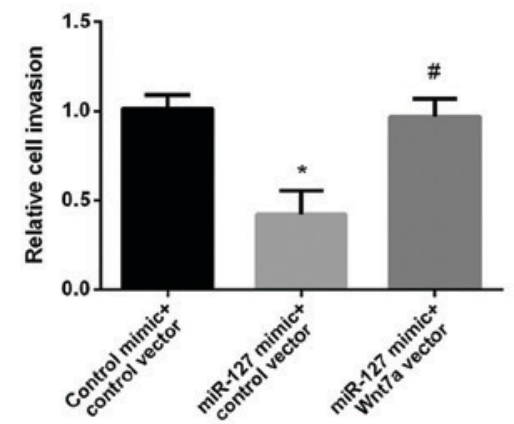

HGC-27

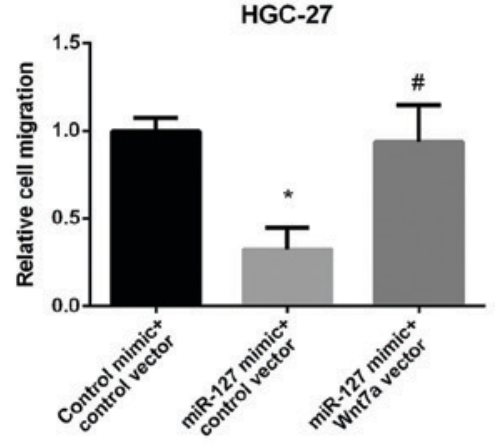

HGC-27

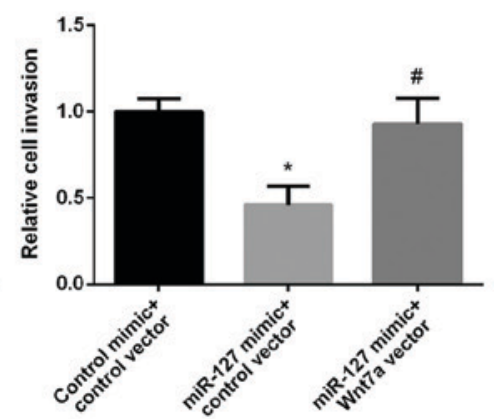

Figure 5. Effect of Wnt7a on gastric cancer (GC) cell migration and invasion regulated by miR-127. Images and quantitative (A) cell migration and (B) cell invasion in GC cells after treatment with miR-127 mimic or both miR-127 mimic and Wnt7a vector evaluated by Transwell migration assays. " $\mathrm{P}<0.05$; ${ }^{\mathrm{P}} \mathrm{P}<0.05$. 
that Wnt7a expression increased in GC and silencing Wnt7a inhibited GC cell migratory and invasive ability.

There are some limitations of this study. Our research did not conduct clinical study. Thus, future clinical study focusing on miR-127/Wnt7a association with clinicopathologic features of human patients, as well as their association with patient prognosis is necessary. In addition, further study of serum expression levels in GC patients may help to develop effective biomarkers for the diagnosis of gastric cancer.

Collectively, miR-127 expression was upregulated while Wnt7a was downregulated in GC. The relationship between miR-127 and Wnt7a expression was negatively correlated. We first proved that Wnt7a was a direct target of miR-127 in the regulation of the progression of GC and Wnt7a could partially reverse the suppression effect of miR-127 in GC, indicating miR-127/Wnt7a axis has a potential application value in GC diagnosis and therapy.

\section{Acknowledgements}

Not applicable.

\section{Funding}

No funding was received.

\section{Availability of data and materials}

The datasets used and/or analyzed during the present study are available from the corresponding author on reasonable request.

\section{Authors' contributions}

LW contributed significantly to data analysis and manuscript preparation. XW performed the data analyses. XJ contributed to the conception of the study. All authors read and approved the final manuscript.

\section{Ethics approval and consent to participate}

The study was approved by the Ethics Committee of Jilin University (Changchun, China). Twenty tumor tissues were obtained from GC patients who underwent surgery at the China-Japan Union Hospital, Jilin University after signing written consent.

\section{Patient consent for publication}

Not applicable.

\section{Competing interests}

The authors declare that they have no competing interests.

\section{References}

1. Ferro A, Peleteiro B, Malvezzi M, Bosetti C, Bertuccio P, Levi F, Negri E, La Vecchia $C$ and Lunet N: Worldwide trends in gastric cancer mortality (1980-2011), with predictions to 2015, and incidence by subtype. Eur J Cancer 50: 1330-1344, 2014.
2. Chen W, Zheng R, Zhang S, Zhao P, Zeng H, Zou X and He J: Annual report on status of cancer in China, 2010. Chin J Cancer Res 26: 48-58, 2014.

3. Zhang J, Song Y, Zhang C, Zhi X, Fu H, Ma Y, Chen Y, Pan F, Wang K, Ni J, et al: Circulating miR-16-5p and miR-19b-3p as two novel potential biomarkers to indicate progression of gastric cancer. Theranostics 5: 733-745, 2015.

4. Di Leva G, Garofalo M and Croce CM: MicroRNAs in cancer. Annu Rev Pathol 9: 287-314, 2014.

5. Kohlhapp FJ, Mitra AK, Lengyel E and Peter ME: MicroRNAs as mediators and communicators between cancer cells and the tumor microenvironment. Oncogene 34: 5857-5868, 2015.

6. Lan H, Lu H, Wang X and Jin H: MicroRNAs as potential biomarkers in cancer: Opportunities and challenges. BioMed Res Int 2015: 125094, 2015.

7. Ge X, Cui H, Zhou Y, Yin D, Feng Y, Xin Q, Xu X, Liu W, Liu S and Zhang Q: miR-320a modulates cell growth and chemosensitivity via regulating ADAM10 in gastric cancer. Mol Med Rep 16: 9664-9670, 2017.

8. Ji FJ, Wu YY, An Z, Liu XS, Jiang JN, Chen FF and Fang XD: Expression of both poly $\mathrm{r}(\mathrm{C})$ binding protein 1 (PCBP1) and miRNA-3978 is suppressed in peritoneal gastric cancer metastasis. Sci Rep 7: 15488, 2017.

9. Yang L, Zhang W, Wang Y, Zou T, Zhang B, Xu Y, Pang T, Hu Q, Chen M, Wang L, et al: Hypoxia-induced miR-214 expression promotes tumour cell proliferation and migration by enhancing the Warburg effect in gastric carcinoma cells. Cancer Lett 414: 44-56, 2018.

10. Sun GL, Li Z, Wang WZ, Chen Z, Zhang L, Li Q, Wei S, Li BW, $\mathrm{Xu} \mathrm{JH}, \mathrm{Chen} \mathrm{L}$, et al: miR-324-3p promotes gastric cancer development by activating Smad4-mediated Wnt/beta-catenin signaling pathway. J Gastroenterol: Nov 4, 2017 (Epub ahead of print). doi: 10.1007/s00535-017-1408-0.

11. Bi L, Yang Q, Yuan J, Miao Q, Duan L, Li F and Wang S: MicroRNA-127-3p acts as a tumor suppressor in epithelial ovarian cancer by regulating the BAG5 gene. Oncol Rep 36: 2563-2570, 2016.

12. Yu Y, Liu L, Ma R, Gong H, Xu P and Wang C: MicroRNA-127 is aberrantly downregulated and acted as a functional tumor suppressor in human pancreatic cancer. Tumour Biol 37: 14249-14257, 2016.

13. Zhang J, Hou W, Chai M, Zhao H, Jia J, Sun X, Zhao B and Wang R: MicroRNA-127-3p inhibits proliferation and invasion by targeting SETD8 in human osteosarcoma cells. Biochem Biophys Res Commun 469: 1006-1011, 2016.

14. Jiang H, Hua D, Zhang J, Lan Q, Huang Q, Yoon JG, Han X, Li L, Foltz G, Zheng S, et al: MicroRNA-127-3p promotes glioblastoma cell migration and invasion by targeting the tumorsuppressor gene SEPT7. Oncol Rep 31: 2261-2269, 2014.

15. Doerks T, Copley RR, Schultz J, Ponting CP and Bork P: Systematic identification of novel protein domain families associated with nuclear functions. Genome Res 12: 47-56, 2002.

16. Cleary AS, Leonard TL, Gestl SA and Gunther EJ: Tumour cell heterogeneity maintained by cooperating subclones in Wnt-driven mammary cancers. Nature 508: 113-117, 2014.

17. Tian J, He H and Lei G: Wnt/3-catenin pathway in bone cancers. Tumour Biol 35: 9439-9445, 2014.

18. Bikkavilli RK, Avasarala S, Van Scoyk M, Arcaroli J, Brzezinski C, Zhang W, Edwards MG, Rathinam MK, Zhou T, Tauler J, et al: Wnt7a is a novel inducer of $\beta$-catenin-independent tumor-suppressive cellular senescence in lung cancer. Oncogene 34: 5406, 2015.

19. Hirata T, Zheng Q, Chen Z, Kinoshita H, Okamoto J, Kratz J, Li H, Lui N, Do H, Cheng T, et al: Wnt7A is a putative prognostic and chemosensitivity marker in human malignant pleural mesothelioma. Oncol Rep 33: 2052-2060, 2015.

20. Kirikoshi $\mathrm{H}$ and Katoh M: Expression of WNT7A in human normal tissues and cancer, and regulation of WNT7A and WNT7B in human cancer. Int J Oncol 21: 895-900, 2002.

21. MacLean JA II, King ML, Okuda H and Hayashi K: WNT7A Regulation by miR-15b in ovarian cancer. PLoS One 11: e 0156109, 2016.

22. Ramos-Solano M, Meza-Canales ID, Torres-Reyes LA, Alvarez-Zavala M, Alvarado-Ruíz L, Rincon-Orozco B, GarciaChagollan M, Ochoa-Hernández AB, Ortiz-Lazareno PC, Rösl F, et al: Expression of WNT genes in cervical cancerderived cells: Implication of WNT7A in cell proliferation and migration. Exp Cell Res 335: 39-50, 2015. 
23. Livak KJ and Schmittgen TD: Analysis of relative gene expression data using real-time quantitative PCR and the 2(-Delta Delta C(T)) Method. Methods 25: 402-408, 2001.

24. Lu M, Ju S, Shen X, Wang X, Jing R, Yang C, Chu H and Cong H: Combined detection of plasma miR-127-3p and HE4 improves the diagnostic efficacy of breast cancer. Cancer Biomark 18: 143-148, 2017.

25. Shi L, Wang Y, Lu Z, Zhang H, Zhuang N, Wang B, Song Z, Chen G, Huang C, Xu D, et al: miR-127 promotes EMT and stem-like traits in lung cancer through a feed-forward regulatory loop. Oncogene 36: 1631-1643, 2017

26. Gao X, Wang X, Cai K, Wang W, Ju Q, Yang X, Wang H and $\mathrm{Wu} \mathrm{H}$ : MicroRNA-127 is a tumor suppressor in human esophageal squamous cell carcinoma through the regulation of oncogene FMNL3. Eur J Pharmacol 791: 603-610, 2016.

27. Tsai KW, Wu CW, Hu LY, Li SC, Liao YL, Lai CH, Kao HW, Fang WL, Huang KH, Chan WC, et al: Epigenetic regulation of miR-34b and miR-129 expression in gastric cancer. Int J Cancer 129: 2600-2610, 2011.
28. Guo LH, Li H, Wang F, Yu J and He JS: The tumor suppressor roles of miR-433 and miR-127 in gastric cancer. Int J Mol Sci 14: 14171-14184, 2013

29. Polakis P: Wnt signaling in cancer. Cold Spring Harb Perspect Biol 4: 4, 2012.

30. Katoh Y and Katoh M: Identification and characterization of rat Wnt6 and Wnt10a genes in silico. Int J Mol Med 15: 527-531, 2005.

31. Kim BK, Kim I and Yoon SK: Identification of miR-199a-5p target genes in the skin keratinocyte and their expression in cutaneous squamous cell carcinoma. J Dermatol Sci 79: 137-147, 2015.

(i) $\Theta$ This work is licensed under a Creative Commons Attribution-NonCommercial-NoDerivatives 4.0 International (CC BY-NC-ND 4.0) License. 